\title{
Analysis of Conformal-Invariant Scattering Amplitudes for the Two-Dimensional World Model
}

\author{
L. CASTELL \\ Max-Planck-Institut für Physik und Astrophysik, München
}

Revised version received January 15, 1970

\begin{abstract}
The explicit form of the "conformal"-invariant "phase shift" analysis in the $s$-channel for a scattering process involving two incoming and two outgoing particles is derived for the two-dimensional world model. The high energy behaviour of the scattering amplitudes is determined completely (up to a constant factor) by the requirement of "conformal" invariance. It is not possible to obtain this high energy limit by neglecting the masses right from the beginning. The main mathematical problem is the determination of the Clebsch-Gordan coefficients for $S U_{0}(1,1)$ in the momentum basis.
\end{abstract}

\section{Introduction}

In a preceding paper [1] we have discussed a method for the calculation of conformal-invariant scattering amplitudes. Contrary to other authors, who have considered conformal-invariant Lagrangian field theories [2] or off-shell amplitudes [3], we deal with on-shell scattering amplitudes. These scattering processes are not restricted to incoming and outgoing mass zero particles, but include particles with mass $0<m<\infty$. In this note we should like to present a slightly different analysis for the two-dimensional world model. We assume that the scattering amplitude is not only invariant under the "Poincaré" group $S O_{0}(1,1) \otimes T_{2}$, but also under the identity component of the spincovering group of the group $\mathrm{SO}_{0}(2,2) / \mathrm{C}_{2}$, which is the analogue in our model to the conformal group of relativistic space-time ${ }^{1}$. The identity component of the spin-covering group is given by $S U_{0}(1,1) \times S U_{0}(1,1)$; and therefore every Clebsch-Gordan coefficient will separate into a product of two coefficients, which depend on $p^{+}=E+p$ and $p^{-}=E-p$, respectively. Only the irreducible unitary representations of the discrete series [4] $\mathscr{D}_{l / 2}^{+}, l=1,2,3 \ldots$ can possibly be used to describe a physical particle. The restriction of these representations with respect to the translational subgroup [5] shows that the spectrum of $p^{+}$for example is

\footnotetext{
${ }^{1}$ We use "conformal" group always in this sense.
} 
given by $0<p^{+}<\infty$ (whereas we have $-\infty<p^{+}<0$ and $-\infty<p^{+}<+\infty$ for the $\mathscr{D}_{l / 2}^{(-)}$and the continuous representations, respectively).

The incoming particles of mass 0 , "spin" $l / 2$, and positive or negative momentum are represented by $\mathscr{D}_{l / 2}^{(+)} \times 1$ and $1 \times \mathscr{D}_{l / 2}^{(+)}$, respectively.

The particles with mass $m \neq 0,\left(0<m^{2}<\infty\right)$ and "helicity" $\frac{1}{2}(l-\lambda)$ are represented by $\mathscr{D}_{l / 2}^{(+)} \times \mathscr{D}_{\lambda / 2}^{(+)}$. (The conformal quantum numbers $l$ and $\lambda$ of the massive particle are determined by the "spins" of the two mass 0 particles, in which it could decay.) Note that the mass of a particle is no longer a Casimir operator but becomes a variable (if $\neq 0$ ). However, only mass ratios and energy ratios have a physical meaning. The main mathematical problem is to calculate the Clebsch-Gordan coefficients of $S U_{0}(1,1)$ for the discrete series in the momentum basis.

\section{The Calculation of the Clebsch-Gordan Coefficients}

The generators of $S U_{0}(1,1)$ can be represented by

$$
P=p, \quad D=i p \frac{\partial}{\partial p}, \quad K=p \frac{\partial^{2}}{\partial p^{2}}-\frac{C}{p},
$$

in the Hilbert space $\int_{0}^{\infty} \psi^{*} \psi \frac{d p}{p}<\infty$, where the Casimir operator $C$ takes the values $C=\frac{l}{2}\left(\frac{l}{2}-1\right) l=1,2,3 \ldots$. We have now to determine the direct product of two irreducible representations [6] $\mathscr{D}_{l_{1} / 2}^{(+)}\left(p_{1}\right) \cdot \mathscr{D}_{l_{2} / 2}^{(+)}\left(p_{2}\right)$. In the product space we introduce the variables $\eta=p_{1}+p_{2}$ and $\xi=\frac{p_{1}-p_{2}}{p_{1}+p_{2}}$. The product space is then defined by

$$
\int \Phi^{*} \Phi \frac{d \eta}{\eta} \frac{2 d \xi}{1-\xi^{2}}<\infty
$$

For the generators we get

$$
P=\eta, \quad D=i \eta \frac{\partial}{\partial \eta}, \quad K=\eta \frac{\partial^{2}}{\partial \eta^{2}}-\frac{C\left(\xi, \frac{\partial}{\partial \xi}\right)}{\eta} .
$$

The Casimir operator $C$ of the product representation is given by

$$
C g(\xi)=-\left[\left(1-\xi^{2}\right) \frac{\partial^{2}}{\partial \xi^{2}}-\frac{2 C_{1}}{1+\xi}-\frac{2 C_{2}}{1-\xi}\right] g(\xi),-1<\xi<+1 .
$$

The condition of self-adjointness [7] of $C$ gives

$$
C=\frac{l}{2}\left(\frac{l}{2}-1\right), \quad l=l_{1}+l_{2}+2 n, \quad n=0,1,2, \ldots .
$$


All elements of the product Hilbert space can therefore be expanded into $\Phi=\sum_{l} a_{l}(\eta) g_{l}(\xi)$, where $\sum_{l} \int a_{l}^{*}(\eta) a_{l}(\eta) \frac{d \eta}{\eta}<\infty$. (There is no degeneracy.)

The orthonormal set $g_{l}(\xi)$ are the square-integrable solutions of (1), with respect to the measure $2 d \xi / 1-\xi^{2}$.

$$
g_{l}^{l_{1}, l_{2}}(\xi)=\left[\frac{1}{N_{l}^{l_{1}, l_{2}}} \frac{p_{1}^{l_{1}} p_{2}^{l_{2}}}{\left(p_{1}+p_{2}\right)^{l_{1}+l_{2}}}\right]^{\frac{1}{2}} P_{\frac{1}{2}\left(l-l_{1}-l_{2}\right)}^{\left(l_{2}-1\right)}\left(\frac{p_{1}-p_{2}}{p_{1}+p_{2}}\right),
$$

where the symmetry between the two representations $l_{1}$ and $l_{2}$ is expressed by the following relation between the Jacobi polynomials $P_{n}^{(\alpha, \beta)}$

$$
P_{n}^{(\alpha, \beta)}(-\xi)=(-1)^{n} P_{n}^{(\beta, \alpha)}(\xi) .
$$

The normalization factor $N$ is given by

$$
\frac{1}{N_{l}^{l_{1}, l_{2}}}=\frac{(l-1) n ! \Gamma\left(n+l_{1}+l_{2}-1\right)}{\Gamma\left(n+l_{1}\right) \Gamma\left(n+l_{2}\right)}, \quad n=\frac{1}{2}\left(l-l_{1}-l_{2}\right) .
$$

From

$$
\left\langle l, p \mid l^{\prime}, p^{\prime}\right\rangle=\delta_{l, l^{\prime}} p \delta\left(p-p^{\prime}\right)
$$

follows finally for the Clebsch-Gordan coefficient

$$
\left\langle l, p \mid l_{1} p_{1} ; l_{2}, p_{2}\right\rangle=g_{l}^{l_{1}, l_{2}}\left(\frac{p_{1}-p_{2}}{p_{1}+p_{2}}\right) p \delta\left(p-p_{1}-p_{2}\right) .
$$

From "Poincaré"- and dilatational invariance follows for (4) only $M\left(p_{1} / p_{2}\right) p \delta\left(p-p_{1}-p_{2}\right)$. The exact form of $M$ is determined by the reduction of certain irreducible representations of the "conformal" group.

\section{The Scattering Amplitudes}

It is now possible to determine the structure of every "conformal"invariant scattering amplitude by a "partial wave" analysis in the $s$-channel. The invariant scattering amplitude is given by

$$
\begin{aligned}
& \left\langle l_{3}^{ \pm}, p_{3}, m_{3} ; l_{4}^{ \pm}, p_{4}, m_{4}|S-1| l_{1}^{ \pm}, p_{1}, m_{1} ; l_{2}^{ \pm}, p_{2}, m_{2}\right\rangle \\
& =F_{l_{1}^{\frac{3}{1}}, l_{2}^{ \pm}}^{l \pm}\left(s, m_{i}\right) \frac{S}{2} \delta\left(E_{1}+E_{2}-E_{3}-E_{4}\right) \delta\left(p_{1}+p_{2}-p_{3}-p_{4}\right),
\end{aligned}
$$

where $s=\left(E_{1}+E_{2}\right)^{2}-\left(p_{1}+p_{2}\right)^{2}$. The one-particle states are defined in the Hilbert space of square-integrable functions of momentum space

$$
\int_{\substack{0<m^{2}<\infty \\ E>0}} \psi^{*} \psi \frac{d E d p}{m^{2} / 2}, \quad \text { and } \int_{\substack{m^{2}=0 \\ E>0}} \psi^{*} \psi \frac{d E}{E}, \quad(p>0 \text { or } p<0),
$$


for the massive and massless case, respectively. We shall determine $F$ for several reactions using the results (3) and (4). $F$ is determined up to constant "phases" $f_{l, \lambda}$, which means for example that the strength of the coupling in a Lagrangian field theory is not determined by conformal invariance.

The scattering amplitude for the most general scattering process with 4 massive particles involved

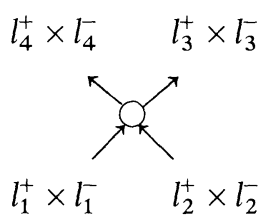

is given by

$$
\begin{aligned}
& F=\sum_{l=\max \left(l_{1}^{+}+l_{2}^{+} ; l_{3}^{+}+l_{4}^{+}\right)}^{\infty} \sum_{\lambda=\max \left(l_{1}^{-}+l_{2}^{-} ; l_{3}^{-}+l_{4}^{-}\right)}^{\infty} g_{l}^{* l_{3}^{+}, l_{4}^{+}}\left(\frac{p_{3}^{+}-p_{4}^{+}}{p_{3}^{+}+p_{4}^{+}}\right) \\
& \cdot g_{\lambda}^{* l_{3}^{-}, l_{4}^{-}}\left(\frac{p_{3}^{-}-p_{4}^{-}}{p_{3}^{-}+p_{4}^{-}}\right)\left(f_{l, \lambda}-1\right) g_{l^{+}}^{l_{1}^{+}, l_{2}^{+}}\left(\frac{p_{1}^{+}-p_{2}^{+}}{p_{1}^{+}+p_{2}^{+}}\right) g_{\lambda}^{l_{1}^{-}, l_{\overline{2}}^{-}}\left(\frac{\overline{p_{1}^{-}-p_{2}^{-}}}{\overline{p_{1}^{-}}+p_{2}^{-}}\right) .
\end{aligned}
$$

The constants $f_{l, \lambda}$ have to be determined by a dynamical theory. From unitary follows $\left|f_{l, \lambda}\right| \leqq 1$.

We shall restrict the physical analysis of (5) to a few remarks. The high energy behaviour of $g_{l}^{l_{1}, l_{2}}(\xi)$ is determined by the first factor of (2), as in the limit $s \rightarrow \infty$ we have $p_{1}^{+} / p_{2}^{+} \rightarrow s / m_{2}^{2}, p_{1}^{-} / p_{2}^{-} \rightarrow m_{1}^{2} / s$, etc.

So the high energy behaviour of the scattering amplitude is fixed completely by the "kinematical" restrictions imposed by "conformal" invariance.

From (5) we obtain the following high energy behaviour (uniform convergence assumed)

$$
F \sim \frac{m_{1}^{l_{1}^{-}} m_{2}^{l^{+}} m_{3}^{l_{3}^{-}} m_{4}^{l_{4}^{+}}}{S^{\frac{1}{2}\left(l_{1}^{-}+l_{2}^{+}+l_{3}^{-}+l_{4}^{+}\right)} .}
$$

If one had no specific information, which irreducible unitary representation of the "conformal" group describes an elementary particle, one can conclude from (5) that $F$ tends to zero at least like $c / s^{2}$, as $l \geqq 1$.

Next we consider the opposite case, the scattering of mass 0 particles.

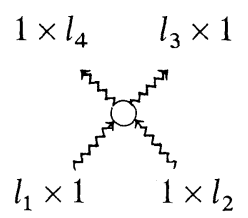


From (3) follows that $F=\left(f_{l_{1}, l_{2}}-1\right) \delta_{l_{1}, l_{3}} \delta_{l_{2}, l_{4}}$. So there could be only diffractive scattering $\left(\left|f_{l_{1}, l_{2}}\right|<1\right)$. Moreover there is only a finite number of inelastic channels. Note that for $l_{1}=l_{2}=1$ (Thirring model) there exists no inelastic channel, i.e. there is no scattering $f_{1,1}=1$. It has been known for the 2-dimensional "conformal" invariant Lagrangian field theory proposed by Thirring in 1957 that there is no "neutrino-neutrino" scattering. Our proof is more general; no renormalization procedure has been used. In the group theoretical framework it is possible to define the incoming or outgoing two particle state unambiguously by the conformal quantum numbers $l_{1}=l_{2}=1$. The state which contains in addition one infrared particle for example can have only the quantum numbers $l_{1}=2,3,4 \ldots, l_{2}=1$, or $l_{1}=1, l_{2}=2,3,4 \ldots$.

The reaction in which one massive and three massless particles participate is of importance for the understanding of the structure of the high energy limit.

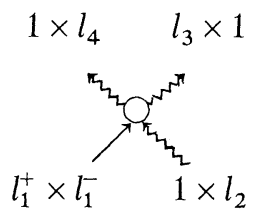

$F$ is given by

$$
F=\frac{f_{l^{+}, l_{4}} \delta_{l_{1}^{+}, l_{3}}}{\left(N_{l_{4}}^{l_{1}, l_{2}}\right)^{\frac{i^{2}}{2}}} \frac{m^{l_{1}^{-}}\left(s-m^{2}\right)^{\frac{l_{2}}{2}}}{s^{\frac{1}{2}\left(l_{1}^{-}+l_{2}\right)}} P_{n}^{l_{2}-1, l_{1}^{-}-1}\left(\frac{2 m^{2}}{s}-1\right), \quad l_{4}=l_{1}^{-}+l_{2}+2 n .
$$

In the limit of high energy we get

$$
F \sim\left(\frac{m^{2}}{s}\right)^{\frac{l_{1}^{-}}{2}} .
$$

The common belief that one can neglect the mass at very high energy and replace it by 0 right from the beginning turns out to be incorrect. The matrix element is tending to zero, and becomes independent of the "spins" of the high energy mass 0 particles. This is apparently the type of simplification which might appear at very high energy.

Finally we consider pair creation from mass 0 particles.

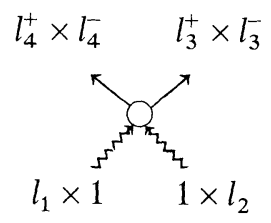


There is also only one "phase" $f_{l_{1}, l_{2}}$ involved. For the high energy limit we obtain

$$
F \sim \frac{m_{3}^{l^{-}} m_{4}^{l_{4}^{+}}}{S^{\frac{1}{2}\left(l_{3}^{-}+l_{4}^{+}\right)}}, \quad l_{1}=l_{3}^{+}+l_{4}^{+}+2 n, \quad l_{2}=l_{3}^{-}+l_{4}^{-}+2 m .
$$

We intend to apply a similar analysis to the 4-dimensional case. The restriction on the high energy limit one will obtain is probably not so stringent as in the 2-dimensional case.

The author would like to thank Prof. W. Heisenberg, Prof. H. P. Dürr, Prof. H. D. Doebner and Dr. P. Breitenlohner for interesting discussions.

Note added in proof. For the conformal-invariant scattering amplitudes one can derive interesting sum rules. For example we obtain for the amplitude on page 131

$$
\int_{m^{2}}^{\infty}\left|F\left(s, m^{2}\right)\right|^{2} \frac{d s}{s-m^{2}}=\left|f_{l_{1}^{+}, l_{4}}\right|^{2} \delta_{l_{1}^{+}, l_{3}} .
$$

\title{
References
}

1. Castell, L.: Integral transformations in momentum space and conformal invariance. M. P. I. Munich, preprint, October 1969, to be published in J. Math. Phys.

2. Gross, D. J., Wess, J.: CERN preprint, TH 1076-CERN (1969).

3. Coon, D. D.: J. Math. Phys. 10, 1314 (1969).

4. Bargmann, V.: Ann. Math. 48, 568 (1947).

5. Castell, L.: ICTP, Trieste, preprint IC/67/71, to be published in J. Math. Phys.

6. Holman, W. J., Biedenharn, L. C.: Ann. Phys. 39, 1 (1966); 47, 205 (1968).

7. Titchmarsh, E. C.: Eigenfunction expansion I. Oxford: Clarendon Press 1962.

Bateman, H.: Higher transcendental functions. Vol. 2. New York: McGraw-Hill Book Company, Inc. 1953.

\author{
L. Castell \\ Max-Planck-Institut \\ für Physık und Astrophysik \\ 8000 München 23 \\ Föhringer Ring 6
}

